\title{
Periprosthetic joint infections after total hip replacement: an algorithmic approach
}

\author{
Mohamed Sukeik ${ }^{1, *}$ and Fares Sami Haddad ${ }^{2}$ \\ ${ }^{1}$ Foothills Medical Centre, 140329 St NW Calgary, AB T2N 2T9, Canada \\ 2 University College London Hospital, 235 Euston Road, London NW1 2BU, UK
}

Received 5 June 2018, Accepted 28 January 2019, Published online 28 February 2019

\begin{abstract}
An algorithm for managing periprosthetic joint infections (PJIs) after total hip replacement (THR) surgery using a multidisciplinary approach and a clearly defined protocol may improve infection eradication rates. In this article, we present an algorithm for the management of different types of PJIs including the acutely infected cemented and cementless THRs where the components are well-fixed postoperatively and when the infection is secondary to haematogenous spread in previously well-functioning and well-fixed implants. For chronic PJIs where the components are often loose, the standard treatment includes a two-stage revision procedure. However, in a highly selected subset of patients, a single-stage approach has been utilised with high rates of eradicating infections.
\end{abstract}

Key words: Periprosthetic, Joint, Infection, Hip replacement, Arthroplasty.

\section{Introduction}

Health services are experiencing an exponential global rise in numbers of lower limb arthroplasty procedures performed for an ageing population. Over the last five years, the UK National Health Service witnessed a growth of hip and knee arthroplasty procedures by $4000-5000$ cases/year [1]. Subsequently, even a minimal prosthetic joint infection (PJI) rate of $0.57 \%$ constitutes a major concern [2], especially with the financial burden of a single revision procedure for sepsis exceeding $£ 21,000$ [3]. The picture is further complicated by the continuous metamorphosis and emergence of new resistant bacterial strains as well as infections with rare organisms [4].

Challenges including diagnostic uncertainty, immunocompromised patients, recurrent infection, infection around a well-fixed implant and substantial bone loss require careful preoperative assessment and well-defined treatment plans [5]. However, there is still no consensus over a standard treatment strategy for PJIs which has accounted for the extensive variability in infection eradication rates in the literature [6,7]. Therefore, an algorithm utilising a multidisciplinary approach and a clearly defined protocol may improve infection rates and contribute to standardising management of PJI after THA.

We present in this study an algorithmic approach to treating different types of PJIs after THA surgery. The protocol involves aggressive surgery removing all mobile and non ingrown parts and exchanging them at the same sitting for acute infection, and

\footnotetext{
*Corresponding author: msukeik@hotmail.com
}

selective single- versus two-stage strategy for established infections based on host, organism and local factors.

\section{Protocol}

In a case of suspected THA infection, the patient should be promptly referred to the specialist hip team which utilises a multi-disciplinary approach in managing such infections as this is a specialised procedure and there is no role for simple incision and drainage or repetitive washouts which result in emergence of resistant microorganisms [5,8]. Clinical presentation (pain, fever, swelling, skin redness, discharging sinus), serologic testing (erythrocyte sedimentation rate $[\mathrm{ESR}]>30 \mathrm{~mm} / \mathrm{h}$; C-reactive protein $[\mathrm{CRP}]>10 \mathrm{mg} / \mathrm{L}$ ), hip aspiration and biopsy with microbiology and cell count analyses help us diagnose PJI [8-10]. Definitive diagnosis however, is established when threeto-six specimens are sampled from different sites at the time of surgery (e.g., capsule, femur and acetabulum) and the same microorganism is cultured from at least two specimens [10-13]. The extent of infection and the interval for which it has been present play a role in the choice of treatment and the chances for successful eradication of infection as follows:

\section{1) Acute infection}

This is defined as an infection occurring within 6-8 weeks of the index operation (primary or revision) or of haematogenous spread from a confirmed source of infection elsewhere 
in a previously well-functioning implant [12,14,15]. In haematogenous infections, a full workup to establish the source of infection should be undertaken preoperatively, including a comprehensive history of recent systemic infections or invasive procedures causing bacteremic seeding of the hip, and investigations should include a throat swab, chest radiograph, and urine, stool and blood cultures [14]. Decision to perform surgery should be based on a high index of suspicion from clinical presentation and serologic testing. Diagnostic aspiration and biopsies in acute infections may delay surgical intervention and also carries variable sensitivity and specificity rates for diagnosing infection $0.50-0.93$ and $0.82-0.97$, respectively [16]. Treatment of acute infection is subdivided according to the type of prosthetic fixation of the original implant:

\section{a) Cemented prostheses}

An aggressive open debridement with exchange of mobile parts and retention of the implant in stable components with no evidence of immunosuppression, and overlying soft tissue and skin of good condition is associated with good results of infection control, especially when the infection is diagnosed within the first week after the index procedure [13-15,17,18]. The aim of rapid intervention with thorough open debridement is to prevent the production of any biofilm by the infecting organism, paramount for successful treatment of infection $[7,19]$. Patients undergo an open complete synovectomy, multiple tissue sampling, exchange of femoral heads and acetabular inserts, debridement of all aspects of the joint, irrigation with solutions such as hydrogen peroxide and Betadine ${ }^{\circledR}$ solutions, and pulsatile lavage $[15,18,20]$.

\section{b) Cementless prostheses}

For acute haematogenous infections in previously wellfunctioning and well-fixed cementless implants, the same protocol for cemented prostheses can be followed as detailed above. However, in acute postoperative infections, once the debridement is complete and samples are sent, another option is to proceed to a single-stage revision procedure where all drapes, gowns, gloves and equipment are changed to create a new, sterile environment. A direct exchange single-stage cementless THA can then be performed as this represents an ideal opportunity to remove both the implant and its biofilm prior to ingrowth [19,21]. Hansen et al. [21] published their series of 27 patients who were treated using this strategy and at a minimum of 27 months reported that 19 of the 27 patients (70\%) retained their implants but four out of those patients required further debridement in order to obtain control of infection.

For both treatment modalities, patients need to continue antibiotic therapy tailored to the sensitivities of intraoperative cultures for at least six weeks until inflammatory markers (CRP, ESR) and the plasma albumin concentration return to within normal limits $[8,14]$. Early conversion to oral antibiotics is dictated by sensitivities and consultation with the microbiology team with whom multidisciplinary meetings should be held on a regular basis $[18,20]$.

\section{2) Chronic infection}

In chronic PJIs, the protocol includes careful assessment of local soft tissues, baseline CRP and ESR, and hip aspiration combined with tissue biopsy as this has shown improved sensitivity and accuracy for diagnosing infection after at least four weeks of discontinuing any antibiotic therapy [18,22]. Plain anteroposterior and lateral radiographs should also be performed, with additional CT if deemed necessary for further acetabular assessment $[8,23,24]$. Once the diagnosis of PJI is suggested by clinical findings and investigations, and the patients are deemed fit and are agreeable to having surgery, patients are considered for either a single- or two-stage revision procedure according to the following:

\section{a) Single-stage revision}

A single-stage revision is carried out under strict conditions including: minimal/moderate bone loss, non-immunocompromised patients, healthy soft tissues, a known organism with known sensitivities, and when appropriate antibiotics are available [23,25-27]. The operation is split into two parts; the first consists of an open aggressive debridement with removal of all components and cement, during which multiple samples are sent to microbiology and irrigation with hydrogen peroxide and Betadine ${ }^{\circledR}$ solutions, and then pulsatile lavage is done. The area is then soaked in aqueous betadine and the wound edges approximated. This is considered to be the end of the first part of the operation and the patient is re-draped and new instruments are used. The surgical team rescrub and put on new gowns [23]. After a further lavage, implantation of a new prosthesis is performed using antibiotic-loaded cement (ALC) or antibiotic-loaded bone graft as needed [18,26,27]. Patients continue antibiotic therapy tailored to the sensitivities of intraoperative cultures for at least six weeks until inflammatory markers (CRP, ESR) and the plasma albumin concentration return to within normallimits. The change from intravenous to oral therapy is effected as soon as full organism sensitivity profile is available [28,29].

\section{b) Two-stage revision}

This is the gold standard for treatment of chronically infected and complex THA infections as the successful eradication of a PJI is over 90\% [24,30-32]. Intraoperatively, the first part of the operation is similar to a single-stage revision. However, after rescrubbing and re-draping, a temporary articulating ALC spacer is implanted instead. This spacer normally contains broad spectrum antibiotics such as vancomycin and gentamicin to cover organisms commonly encountered with deep periprosthetic infections whilst reducing the development of resistant strains [18,33]. Postoperatively, the patient is allowed to mobilise partial weight-bearing with crutches and is discharged home when deemed safe. Antibiotic therapy tailored to the sensitivities of intraoperative cultures is continued for 4-6 weeks $[18,24,29]$. The decision to proceed with insertion of a new prosthesis is determined by the clinical response of the patient including wound healing, inflammatory and nutritional markers indicating resolution of infection together with 
Table 1. Previous studies reporting PJI eradication rates following various treatment strategies.

\begin{tabular}{|c|c|c|c|c|c|c|}
\hline Author & Treatment & $\begin{array}{l}\text { Infection } \\
\text { site }\end{array}$ & $\begin{array}{l}\text { Number } \\
\text { of cases }\end{array}$ & $\begin{array}{l}\text { Exchange of } \\
\text { mobile parts }\end{array}$ & $\begin{array}{c}\text { Eradication } \\
\text { rate }(\%)\end{array}$ & $\begin{array}{c}\text { Follow-up } \\
\text { in years }\end{array}$ \\
\hline Aboltins et al. [48] & Debridement & Hip/Knee & 17 & Yes & 88.2 & 2.3 \\
\hline Klouche et al. [57] & Debridement & Hip & 12 & Partly & 75 & 3.3 \\
\hline Krasin et al. [60] & Debridement & Hip & 7 & No & 71 & 2.5 \\
\hline Martinez-Pastor et al. [56] & Debridement & Hip/Knee & 47 & Yes & 74.5 & 1.2 \\
\hline Sukeik et al. [14] & Debridement & Hip & 26 & Yes & 77 & 6.6 \\
\hline Choi et al. [46] & Single & Hip & 17 & - & 82 & 5.2 \\
\hline Klouche et al. [57] & Single & Hip & 38 & - & 100 & 2 \\
\hline Winkler et al. [45] & Single & Hip & 37 & - & 92 & 4.4 \\
\hline Zeller et al. [58] & Single & Hip & 157 & - & 95 & 5 \\
\hline Oussedik et al. [54] & Single & Hip & 11 & - & 100 & 6.8 \\
\hline Berend et al. [30] & Two-stage & Hip & 186 & - & 83 & 4.4 \\
\hline Klouche et al. [57] & Two-stage & Hip & 46 & - & 98 & $>2$ \\
\hline Masri et al. [59] & Two-stage & Hip & 29 & - & 90 & $>2$ \\
\hline Ibrahim et al. [34] & Two-stage & Hip & 125 & - & 96 & $>5$ \\
\hline
\end{tabular}

performing a further aspiration which is negative [8,18,24]. At the second stage, the spacer is removed and the underlying cement mantle is fragmented and removed piecemeal, without sacrificing bone stock. Appropriate implants are then reimplanted with either cemented or cementless components, and allografts may be used in cases of severe bone loss. The importance of a multi-disciplinary team approach as well as strict adherence to the above protocol is important in order to achieve high rates of infection control [25,29,34].

Regardless of the treatment strategy followed, all patients should be followed up postoperatively at two and six weeks, six months, one year, and then on a yearly basis, looking for clinical symptoms and signs of infection, as well as CRP and ESR level testing. Plain radiographs including an AP pelvis and lateral of both hips should be requested at every followup appointment. Stem position, radiolucencies and osteolysis should be assessed. The stem angle is classified as neutral, varus or valgus. A stem angle is considered neutral if its axis is within $2^{\circ}$ of the femoral shaft axis. Femoral and acetabular radiolucencies are classified according to Gruen et al. [35] and DeLee and Charnley [36] zones, respectively. Loosening is diagnosed if the radiolucent zone around one or both components is $2 \mathrm{~mm}$ or more in width and a patient has symptoms on weightbearing and motion that are relieved by rest [37]. Osteolytic lesions are documented and classified on the basis of their size (linear or expansile) and their location according to previously published criteria by Zicat et al. [38]. Of note, though, is that substantial interobserver variability can be expected using these systems [39,40]. Definition of eradication of infection has been variable in the literature, but mostly includes the absence of clinical, serologic, and radiographic signs of infection and absence of death secondary to infection or treatment during the follow-up period. Failure on the other hand includes any major operation performed in any subgroup of patients for eradication of infection, including a two-stage revision, excision arthroplasty, arthrodesis and amputation, or the need for long-term antibiotic suppression. A reinfection is considered to be an infection with the same or another organism.

\section{Discussion}

Despite the relatively low rates of PJIs after THAs, they remain a leading cause of revision surgery due to an everincreasing number of hip arthroplasties performed yearly for an ageing population [8]. Difficulties with reaching a consensus on what defines infection and which strategy best eradicates it led to extensive variability in infection rates in the literature, until recent efforts from the International consensus meeting on managing PJIs defined what constitutes an infection [12]. Specialist tertiary centres dealing with PJIs on a regular basis may improve infection-free survival and contribute to a global approach for managing PJI. Therefore, we aimed in this study at presenting our preferred algorithm for treating PJIs after THA surgery.

Results for eradication of infection using an aggressive early debridement and exchange of mobile parts for acute infections as detailed here and two-stage revision for chronic infections where a clear protocol has been followed yield high rates of eradicating PJIs (Table 1) [41-43]. It is of note, though, that the inclusion and exclusion criteria, as well as management protocols, varied among studies reported in the literature, occasionally including all types of periprosthetic infections rather than acute or chronic infections only. Additionally, some of the studies did not differentiate between hips and knees when reporting their findings which resulted in a wide range of eradicating infection (Table 1).

On the other hand, single-stage revisions for chronic infections are regaining momentum, and studies reporting high rates of eradicating infection certainly reflect a strict protocol followed similar to the one described in this review [18,44,45]. Single-stage direct exchange protocol for acutely infected cementless THAs remains a novel approach which has not yet gained popularity, but presents a time-limited opportunity to remove the implants prior to ingrowth in a cementless THA [21]. In comparison with aggressive debridement with exchange of mobile parts in cemented THAs [14], it showed almost similar results for eradication of infection (70\% vs. $77 \%$ ) with a single operation, whereas a few of the cases in the debridement group 
required several wash outs with the additional soft tissue trauma caused before eradication of infection [21].

A number of challenging cases can add to the complexity of dealing with PJIs. For example, culture negative infections, resistant organisms, reconstruction of large bony defects after removal of autologous or allogeneic bone grafts used in primary operations for hip dysplasia or revision surgery and dealing with metal on metal bearings and dual mobility implants. Principles for treating such difficult cases remain the same but with special considerations for each case. For example, in culture negative and resistant organisms, diagnosis can be challenging, but once established using all the diagnostic tools available and applying the International Consensus Meeting (ICM) on management of PJIs diagnostic criteria [12], treatment strategies should follow a two-stage reimplantation procedure as debridement and exchange of mobile parts and single-stage revisions are associated with higher failure rates [18,46-49]. Patients who fail treatment may require salvage procedures such as long-term antibiotics, resection arthroplasty, fusion and amputation [50].

Patients who had undergone autologous or allogeneic bone grafting as part of their primary operations for hip dysplasia or revision surgery constitute another challenging group of patients. As there is limited evidence in the literature guiding treatment of these groups of patients, it is our preference that if such bone grafts have osseointegrated and it has been a number of years since the primary operation with no evidence of an underlying chronic infection that there is no need to remove those grafts. Otherwise, treatment for the different types of PJIs encountered remains the same as detailed in the above protocol. We also prefer removing any underlying metalwork from the primary procedure including screws utilised for the original fixation of the cup if it is safe and feasible to do so, as there is evidence that retained metalwork may contribute to incomplete debridement and possible recurrence of the infection [18,20].

Metal on metal bearings constitute another challenge due to the difficulty of establishing the correct diagnostic thresholds for inflammatory markers in the serum and synovial fluid samples. In fact, a number of studies suggested that CRP and ESR are not reliable alone in diagnosing PJI in metal on metal cases and that the synovial fluid WBC count can frequently be falsely positive and, therefore, should be relied on only if a manual count is done and a differential can be performed [12,51,52]. The ICM also suggests being cautious whilst applying its diagnostic criteria for PJIs in metal on metal cases for the same reasons [12].

Dual mobility bearings have been associated with lower dislocation rates and no increase in complications when compared with other THA bearings. Infections in particular are not higher with dual mobility bearings. Therefore, management of PJIs in this context has not been widely discussed in the literature. However, in the few published articles a number of points should be noted including the fact that diagnosis of PJI can be challenging, especially in the setting of intraprosthetic dislocation due to associated metal debris, and the principles should follow the above recommendations in those cases $[12,18]$. In the cases of acute infection, shell stability should be assessed intraoperatively as it is easy to revise it in the early postoperative phase [53]. Additionally, dual mobility bearings allow easier exchange of the liner, but the head requires removal which may risk damage to the taper. In chronic cases, a two-stage revision procedure remains the gold standard. However, single-stage revision may still be considered in a strictly selected group of patients as detailed previously [53].

\section{Conclusion}

In conclusion, we present a clear protocol for treating periprosthetic hip arthroplasty infections which has been supported by a number of studies in the literature from centres dealing with PJIs on a regular basis, yielding high rates of eradicating infection. We also agree that only through the use of standardised terminology that an international language of comparative results will be feasible and, therefore, we support efforts made to standardise the definition of PJI $[10,13,54,55]$. However, in view of the heterogeneity of clinical presentation and variability of diagnostic tests' validity and reliability in diagnosing infection, the debate for a common strategy of treatment has yet to be finalised.

\section{Conflict of interest}

Authors certify that they have no financial conflict of interest.

\section{References}

1. NJR (2017) Available at http://www.njrreports.org.uk/Portals/0/ PDFdownloads/NJR\%2014th\%20Annual\%20Report\%202017. pdf.

2. Phillips JE, Crane TP, Noy M, Elliott TS, Grimer RJ (2006) The incidence of deep prosthetic infections in a specialist orthopaedic hospital: a 15-year prospective survey. J Bone Joint Surg Br 88, 943-948.

3. Vanhegan IS, Malik AK, Jayakumar P, Ul Islam S, Haddad FS (2012) A financial analysis of revision hip arthroplasty: the economic burden in relation to the national tariff. J Bone Joint Surg Br 94, 619-623.

4. Eid AJ, Berbari EF, Sia IG, Wengenack NL, Osmon DR, Razonable RR (2007) Prosthetic joint infection due to rapidly growing mycobacteria: report of 8 cases and review of the literature. Clin Infect Dis 45, 687-694.

5. Haddad FS, Masri BA, Garbuz DS, Duncan CP (1999) The treatment of the infected hip replacement. The complex case. Clin Orthop Relat Res 144-156.

6. Kaltsas DS (2004) Infection after total hip arthroplasty. Ann R Coll Surg Engl 86, 267-271.

7. Moyad TF, Thornhill T, Estok D (2008) Evaluation and management of the infected total hip and knee. Orthopedics 31, 581-588; quiz 9-90.

8. Sukeik MTS, Haddad FS (2009) Management of periprosthetic infection in total hip arthroplasty. Orthop Trauma 23, 342-349.

9. Parvizi J, Gehrke T (2014) Definition of periprosthetic joint infection. J Arthroplasty 29, 1331.

10. Shohat N, Bauer T, Buttaro M, et al. (2019) Hip and knee section, what is the definition of a periprosthetic joint infection 
(PJI) of the knee and the hip? Can the same criteria be used for both joints? Proceedings of international consensus on orthopedic infections. J Arthroplasty 34(2S), S325-S327.

11. Atkins BL, Athanasou N, Deeks JJ, et al. (1998) Prospective evaluation of criteria for microbiological diagnosis of prosthetic-joint infection at revision arthroplasty. The OSIRIS Collaborative Study Group. J Clin Microbiol, 36 2932-2939.

12. AbdelKarim M, Andrawis J, Bengoa F, et al. (2019) Hip and knee section, diagnosis, algorithm: Proceedings of international consensus on orthopedic infections. J Arthroplasty 34(2S), S339-S350.

13. Parvizi J, Gehrke T, Mont MA, Callaghan JJ (2019) Introduction: Proceedings of international consensus on orthopedic infections. J Arthroplasty 34(2S), S1-S2.

14. Sukeik M, Patel S, Haddad FS (2012) Aggressive early debridement for treatment of acutely infected cemented total hip arthroplasty. Clin Orthop Relat Res 470, 3164-3170.

15. Volpin A, Sukeik M, Alazzawi S, Haddad FS (2016) Aggressive early debridement in treatment of acute periprosthetic joint infections after hip and knee replacements. Open Orthop J 10, 669-678.

16. Spangehl MJ, Masri BA, O’Connell JX, Duncan CP (1999) Prospective analysis of preoperative and intraoperative investigations for the diagnosis of infection at the sites of two hundred and two revision total hip arthroplasties. J Bone Joint Surg Am 81, 672-683.

17. Davis JS (2005) Management of bone and joint infections due to Staphylococcus aureus. Intern Med J 35(Suppl 2), S79-S96.

18. Bori G, Kelly M, Kendoff D, et al. (2019) Hip and knee section, treatment, prosthesis factors: Proceedings of international consensus on orthopedic infections. J Arthroplasty 34(2S), S453-S457.

19. Mirza YH, Tansey R, Sukeik M, Shaath M, Haddad FS (2016) Biofilm and the role of antibiotics in the treatment of periprosthetic hip and knee joint infections. Open Orthop J 10, 636-645.

20. Abblitt WP, Ascione T, Bini S, et al. (2019) Hip and knee section, outcomes: Proceedings of international consensus on orthopedic infections. J Arthroplasty 34(2S), S487-S495.

21. Hansen E, Tetreault M, Zmistowski B, et al. (2013) Outcome of one-stage cementless exchange for acute postoperative periprosthetic hip infection. Clin Orthop Relat Res 471, 3214-3222.

22. Meermans G, Haddad FS (2010) Is there a role for tissue biopsy in the diagnosis of periprosthetic infection? Clin Orthop Relat Res 468, 1410-1417.

23. Haddad FS, Sukeik M, Alazzawi S (2015) Is single-stage revision according to a strict protocol effective in treatment of chronic knee arthroplasty infections? Clin Orthop Relat Res 473, 8-14.

24. Sukeik M, Haddad FS (2009) Two-stage procedure in the treatment of late chronic hip infections-spacer implantation. Int J Med Sci 6, 253-257.

25. Oussedik SI, Dodd MB, Haddad FS (2010) Outcomes of revision total hip replacement for infection after grading according to a standard protocol. J Bone Joint Surg Br 92, 1222-1226.

26. Zahar A, Gehrke TA (2016) One-stage revision for infected total hip arthroplasty. Orthop Clin North Am 47, 11-18.

27. Nguyen M, Sukeik M, Zahar A, Nizam I, Haddad FS (2016) One-stage exchange arthroplasty for periprosthetic hip and knee joint infections. Open Orthop J 10, 646-653.

28. Sukeik M, Haddad FS (2009) Management of periprosthetic infection in total hip arthroplasty. Orthop Trauma 23, 342-349.
29. Kini SG, Gabr A, Das R, Sukeik M, Haddad FS (2016) Twostage revision for periprosthetic hip and knee joint infections. Open Orthop J 10, 579-588.

30. Berend KR, Lombardi AV Jr, Morris MJ, Bergeson AG, Adams JB, Sneller MA (2013) Two-stage treatment of hip periprosthetic joint infection is associated with a high rate of infection control but high mortality. Clin Orthop Relat Res 471, 510-518.

31. Cooper HJ, Della Valle CJ (2013) The two-stage standard in revision total hip replacement. Bone Joint J 95-B, 84-87.

32. Bejon P, Berendt A, Atkins BL, et al. (2010) Two-stage revision for prosthetic joint infection: predictors of outcome and the role of reimplantation microbiology. J Antimicrob Chemother 65, 569-575.

33. Anagnostakos K, Furst O, Kelm J (2006) Antibiotic-impregnated PMMA hip spacers: Current status. Acta Orthop 77, 628-637.

34. Ibrahim MS, Raja S, Khan MA, Haddad FS (2014) A multidisciplinary team approach to two-stage revision for the infected hip replacement: a minimum five-year follow-up study. Bone Joint J 96-B, 1312-1318.

35. Gruen TA, McNeice GM, Amstutz HC (1979) "Modes of failure" of cemented stem-type femoral components: a radiographic analysis of loosening. Clin Orthop Relat Res 141, 17-27.

36. DeLee JG, Charnley J (1976) Radiological demarcation of cemented sockets in total hip replacement. Clin Orthop Relat Res 121, 20-32.

37. Harkness JW, Crockarell JR (2008) Arthroplasty of the hip. In: Campbell's Operative Orthopaedics. 11th edn, Vol. 1. Canale ST, Beaty JH, Editors. Philadelphia, PA, Mosby, p. 427.

38. Zicat B, Engh CA, Gokcen E (1995) Patterns of osteolysis around total hip components inserted with and without cement. J Bone Joint Surg Am 77, 432-439.

39. McCaskie AW, Brown AR, Thompson JR, Gregg PJ (1996) Radiological evaluation of the interfaces after cemented total hip replacement. Interobserver and intraobserver agreement. J Bone Joint Surg Br 78, 191-194.

40. Kneif D, Downing M, Ashcroft GP, et al. (2005) Peri-acetabular radiolucent lines: inter- and intra-observer agreement on postoperative radiographs. Int Orthop 29, 152-155.

41. Marculescu CE, Berbari EF, Hanssen AD, et al. (2006) Outcome of prosthetic joint infections treated with debridement and retention of components. Clin Infect Dis 42, 471-478.

42. Aboltins CA, Page MA, Buising KL, et al. (2007) Treatment of staphylococcal prosthetic joint infections with debridement, prosthesis retention and oral rifampicin and fusidic acid. Clin Microbiol Infect 13, 586-591.

43. Toulson C, Walcott-Sapp S, Hur J, et al. (2009) Treatment of infected total hip arthroplasty with a 2-stage reimplantation protocol: update on "our institution's" experience from 1989 to 2003. J Arthroplasty 24, 1051-1060.

44. Joulie D, Girard J, Mares O, et al. (2011) Factors governing the healing of Staphylococcus aureus infections following hip and knee prosthesis implantation: a retrospective study of 95 patients. Orthop Traumatol Surg Res 97, 685-692.

45. Winkler H, Stoiber A, Kaudela K, Winter F, Menschik F (2008) One stage uncemented revision of infected total hip replacement using cancellous allograft bone impregnated with antibiotics. J Bone Joint Surg Br 90, 1580-1584.

46. Choi HR, Kwon YM, Freiberg AA, Malchau H (2013) Comparison of one-stage revision with antibiotic cement versus two-stage revision results for infected total hip arthroplasty. J Arthroplasty 28, 66-70. 
47. McLawhorn AS, Nawabi DH, Ranawat AS (2016) Management of resistant, atypical and culture-negative periprosthetic joint infections after hip and knee arthroplasty. Open Orthop J 10, 615-632.

48. Aboltins CA, Dowsey MM, Buising KL, et al. (2011) Gramnegative prosthetic joint infection treated with debridement, prosthesis retention and antibiotic regimens including a fluoroquinolone. Clin Microbiol Infect 17, 862-867.

49. Leonard HA, Liddle AD, Burke O, Murray DW, Pandit H (2014) Single- or two-stage revision for infected total hip arthroplasty? A systematic review of the literature. Clin Orthop Relat Res 472, 1036-1042.

50. Mahmoud SS, Sukeik M, Alazzawi S, Shaath M, Sabri O (2016) Salvage procedures for management of prosthetic joint infection after hip and knee replacements. Open Orthop J 10, 600-614.

51. Yi PH, Cross MB, Moric M, et al. (2015) Do serologic and synovial tests help diagnose infection in revision hip arthroplasty with metal-on-metal bearings or corrosion? Clin Orthop Relat Res 473, 498-505.

52. Saleh A, George J, Faour M, Klika AK, Higuera CA (2018) Serum biomarkers in periprosthetic joint infections. Bone Joint Res 7, 85-93.

53. Hernigou P, Dubory A, Potage D, Roubineau F, Flouzat Lachaniette CH (2017) Dual-mobility arthroplasty failure: a rationale review of causes and technical considerations for revision. Int Orthop 41, 481-490.

54. Oussedik S, Gould K, Stockley I, Haddad FS (2012) Defining peri-prosthetic infection: do we have a workable gold standard? J Bone Joint Surg Br 94, 1455-1456.

55. Workgroup (2011) New definition for periprosthetic joint infection. J Arthroplasty 26, 1136-1138.

56. Martinez-Pastor JC, Munoz-Mahamud E, Vilchez F, et al. (2009) Outcome of acute prosthetic joint infections due to gram-negative bacilli treated with open debridement and retention of the prosthesis. Antimicrob Agents Chemother 53(11), 4772-4777.

57. Klouche S, Leonard P, Zeller V, et al. (2012) Infected total hip arthroplasty revision: one- or two-stage procedure? Orthop Traumatol Surg Res 98(2), 144-150.

58. Zeller V, Lhotellier L, Marmor S, et al. (2014) One-stage exchange arthroplasty for chronic periprosthetic hip infection: results of a large prospective cohort study. J Bone Joint Surg Am 96(1), e1.

59. Masri BA, Panagiotopoulos KP, Greidanus NV, et al. (2007) Cementless two-stage exchange arthroplasty for infection after total hip arthroplasty. J Arthroplasty 22(1), 72-78.

60. Krasin E, Goldwirth M, Hemo Y, Gold A, Herling G, Otremski I (2001) Could irrigation, debridement and antibiotic therapy cure an infection of a total hip arthroplasty? J Hosp Infect 47(3), 235-238. 